\title{
El contexto histórico y cultural y su influencia en la alfabetización mediática
}

\author{
Isamélia Santos Guimarães Carvalho ${ }^{1}$
}

Juliana Guimarães Faria ${ }^{2}$

\section{Resumen:}

Este artículo tiene como objetivo reflexionar sobre la importancia del contexto cultural e histórico en la formación del sujeto y la construcción de su subjetividad, bien como su influencia en el proceso de alfabetización mediática y su acción transformadora en la sociedad. Incorpora los conceptos de la psicología sociohistórica y los postulados teóricos de Vygotsky. Es un estudio teórico, basado en Bock, Furtado y Teixeira (2002), Teixeira (2002), Vygotsky (1995), Hernández (2006), González Rey (1997), Tejedor y Perceval (2008) y Pérez Tornero (2000). Los conceptos se articulan a partir del proceso de construcción del conocimiento y la alfabetización mediática, teniendo en cuenta la cultura de este nuevo contexto mediático y las demandas de la sociedad contemporánea. En conclusión, comprendemos que la "alfabetización mediática" es un concepto subjetivo que ultrapasa la dimensión técnica y operativa, involucrando los aspectos cognitivos y metacognitivos del sujeto, así como el contexto cultural en el que se está insertado.

Palabras clave: Alfabetización mediática, contexto sociohistórico, cultura, Vygotsky.

\section{The historical and cultural context and its influence on media literacy}

\section{Abstract:}

The objective of this article is to reflect on the importance of the cultural and historical context in the mental development of the subject, as well as the construction of his/her subjectivity, influence in the process of media literacy and its transforming capability over the society. Incorporated in this work are the concepts of social- historic psychology and the theoretical postulates of Vygotsky. It is a theoretical study, based on Bock, Furtado and Teixeira (2002), Teixeira (2002), Vygotsky (1995), Hernández (2006), Gonzalez Rey (1997), Tejedor and Perceval (2008) and Pérez Tornero (2000). The concepts are articulated around the process of knowledge construction and media literacy, accounting for the factor of culture in the new media context and the demands of contemporary society. In conclusion, it is understood that "media literacy" is a subjective concept, bypassing the technical and operational dimensions by involving cognitive and metacognitive aspects of the subject, as well as the cultural context surrounding him/her.

Key words: Media literacy, historical and cultural context, culture, Vygotsky.

1 Máster en Comunicación y Educación, por la Universidad Autónoma de Barcelona (UAB) y Doctoranda en Educación por la Facultad de Ciencias de la Educación - Universidad Autónoma de Barcelona. E-mail: isacarvalho27@gmail.com

2 Doutora em Educação pela Universidade Federal de Goiás (UFG). Professora na Faculdade de Letras da Universidade Federal de Goiás (FL/UFG). E-mail: julianagf@ufg.br. 


\section{O contexto histórico e cultural e sua influência na alfabetização midiática}

\section{Resumo:}

Este artigo tem como objetivo refletir sobre a importância do contexto cultural e histórico na formação do sujeito e a construção de sua subjetividade, bem como sua influência no processo de alfabetização midiática e sua ação transformadora na sociedade. Incorpora os conceitos da psicologia socio-histórica e os postulados teóricos de Vygotsky. É um estudo teórico, baseado em Bock, Furtado e Teixeira (2002), Teixeira (2002), Vygotsky (1995), Hernández (2006), González Rey (1997), Tejedor y Perceval (2008) e Pérez Tornero (2000). Os conceitos se articulam a partir do processo de construçáo do conhecimento e da alfabetização midiática, tendo em conta a cultura deste novo contexto midiático e as demandas da sociedade contemporânea. Em conclusão, compreendemos que a "alfabetização midiática” é um conceito subjetivo que ultrapassa a dimensáo técnica e operativa, envolvendo os aspectos cognitivos e metacognitivos do sujeito, assim como o contexto cultural no qual está inserido.

Palavras chave: Alfabetização midiática, contexto socio-histórico, cultura, Vygotsky.

\section{Introducción}

La sociedad de la información y del conocimiento es un hito en la posmodernidad y en todos los cambios que atraviesan este proceso. Entre ellos destacamos la alfabetización mediática y las herramientas, habilidades y competencias necesarias para su desarrollo.

La entrada de las TICs (Tecnologías de la Información y la Comunicación) en la sociedad y su desarrollo acelerado ofrecen una base para el crecimiento de esta sociedad y despierta la necesidad de construir habilidades y competencias para los sujetos que utilizarán esas tecnologías y serán los protagonistas de esta nueva sociedad y sus exigencias. Ese proceso de construcción de habilidades y competencias nos permite mirar, investigar y trabajar con las tecnologías, actuando de forma crítica, articulada con el contexto cultural y los distintos conocimientos del mundo.

El término "alfabetización mediática" hace referencia a una dimensión educativa (alfabetización) y tecnológica (medios). El concepto de alfabetización está vinculado a las referencias de la lectura y la escritura, así como el proceso de aprendizaje. Por ello, se puede aplicar el significado y el sentido del término "Alfabetización" en los medios digitales con la misma importancia y relevancia con que aplicamos en la educación formal, visto que es un proceso de construcción de conocimiento y de adquisición de habilidades y competencias. 
En diferentes contextos encontramos los medios vinculados a los estudios de alfabetización, en que ambos hacen parte de un eje en que la prioridad es que se correspondan con las dimensiones: individuo/construcción, técnico y tecnológico e instrumental y cultural. Actualmente, otras definiciones y conceptos se agregan a esos estudios, las cuales podemos destacar la concepción de la alfabetización mediática como una competencia, donde el sujeto opera cognitivamente y metacognitivamente a través de la tecnología, sus artefactos e infinitas posibilidades y articulaciones.

Este artículo tiene como objetivo hacer una reflexión sobre el pensamiento sociohistórico, el contexto cultural y de cómo esos aspectos se articulan e influyen en el proceso de la alfabetización mediática. Además, comprendemos que el contexto escolar no puede aislarse de esa realidad emergente e inminente. Siendo así, disertaremos acerca del abordaje sociohistórico y enseguida articulamos con el contexto cultural y la alfabetización mediática.

\section{La perspectiva sociohistórica}

El contexto cultural e histórico está subyacente al desarrollo humano y es un aspecto de extrema importancia en la constitución del sujeto, pues ejerce gran influencia en sus opciones, actitudes y actuación en la sociedad. Muchos autores (BOCK; FURTADO; TEIXEIRA, 2002; VYGOTSKY, 1995; HERNANDEZ, 2006) y corrientes de la psicología discuten estas cuestiones y su poder en numerosos aspectos del desarrollo y del aprendizaje, así como en la relación intrínseca que hay entre ambos.

A partir de los estudios de la psicología sociohistórica y su postulado teórico, podemos entender de una manera científica, social, colectiva e histórica, la relación entre el hombre y la sociedad. En este sentido, y vinculando estos conceptos a la Sociedad de la Información y del Conocimiento, podemos darnos cuenta de las influencias de estos aspectos en la alfabetización mediática, en la interacción del individuo con los distintos medios mediáticos y su posicionamiento frente al entorno en que está insertado.

Por lo tanto, pasaremos a revisar algunos conceptos y estudios de la psicología sociohistórica, que son claves para entender las distintas concepciones que conforman el contexto histórico y cultural, así como su relación con el desarrollo, el aprendizaje, los medios y la alfabetización mediática.

La psicología sociohistórica se ha constituido, fundamentalmente, por la crítica a la visión liberal del hombre, en la que defiende la idea del hombre 
como un ser autónomo, responsable de su propio proceso de individuación, en una relación de antagonismo entre el hombre y la sociedad, en que ésta se opone a sus deseos naturales (BOCK; FURTADO; TEIXEIRA, 2002).

La visión del fenómeno psicológico, que es tomado como una entidad abstracta,tiene por naturaleza, características positivas que se manifiestan solamente si sufren impedimentos al mundo social y material. Es decir, considera que estas concepciones liberales construyeron una ciencia en que el mundo psicológico cambió de social y material, pasando luego a definirse de manera abstracta como algo que ya estaba en el hombre, dispuesto a desarrollarse.

Esta mirada liberal naturalizó el mundo psicológico, quitando de la psicología reflexiones sobre el mundo social, en otras palabras, consideran que las cuestiones de carácter histórico y social no deberían vincularse a la psicología. Además, la concepción liberal está vinculada a la visión del individuo autónomo, que no es aceptado por la psicología sociohistórica, pues la misma defiende la premisa básica que el individuo se construye a lo largo de su vida bajo la influenciadel entorno en lo cual está insertado, en las relaciones sociales y culturales establecidas que son decisivas para su individualidad y subjetividad.

En este sentido, no tenemos un desarrollo independiente o previsto por la genética. La condición humana es primordial y sine qua non para nuestra constitución como sujeto, estamos inmersos y sometidos al contexto sociohistórico y al grupo cultural en lo cual estamos insertados.

La acción del hombre sobre la realidad que necesariamente ocurre en la sociedad es un proceso histórico. Y una acción de transformación de la naturaleza que conduce a la transformación del hombre a sí mismo. Cuando produce los bienes necesarios para satisfacer sus necesidades, el hombre establece nuevos parámetros en su relación con la naturaleza, que genera nuevas necesidades, que, a su vez, deben cumplirse. Las relaciones sociales en que se produce en ese proceso se cambian en la medida que se desarrollan las necesidades humanas y producción que pretende satisfacerlas. Es un proceso de transformación constante de las necesidades y la actividad de los hombres y de las relaciones que ellos establecen entre sí para la producción de su existencia. Este movimiento se basa en la contradicción: el desarrollo de las necesidades humanas y las formas de satisfacerlas, y que sólo son posibles a partir de determinadas relaciones sociales, provocan la necesidad de la transformación de estas mismas relaciones y condicionan la aparición de nuevas relaciones sociales. Ese proceso histórico es construido por el hombre y es este proceso histórico que construye al hombre. (BOCK; FURTADO; TEIXEIRA, 2002, p. 117). 
Así pues, atravesamos ese proceso y nos hacemos sujetos en esa travesía donde estamos inmersos en la sociedad, la cultura y la historia, sometidos a la apropiación del mundo, convirtiendo el mundo exterior en un mundo interno, desarrollando de manera singular nuestra individualidad y subjetividad. Así, el hombre se desarrolla, se subjetiva, se individualiza y se convierte en hombre a través de la mediación de las relaciones sociales y de las actividades que realiza, a partir de las ideas y los conceptos del grupo cultural en el cual está insertado.

Vygotsky (1995) retoma ese tema al vincular los procesos psicológicos y el desarrollo del sujeto al contexto histórico y social, definiendo el hombre como histórico y social, no siendo disociado, bajo ninguna hipótesis, de estas cuestiones y de la cultura en ningún punto de su desarrollo.

Según Lev Seminovch Vygotsky(1995), el desarrollo humano está marcado por el entorno histórico y social del sujeto y de la cultura que subyaz ese entorno, es decir, el hombre es constituido y formado por el contexto cultural que lo compone, su historia y el entorno social, siendo un producto de este conjunto de aspectos. Por lo cual, conviene subrayar que el desarrollo humano y sus diferentes caminos son influenciados por la cultura y por la inclusión del sujeto en un determinado grupo cultural.

Para comprender mejor esa influencia, es importante tener una mirada distinta acerca del hombre, su historia, su recogido y actuación en la sociedad y en el mundo. El pensamiento de Vygotsky tuvo gran influencia del Marxismo y los postulados básicos del materialismo dialéctico e histórico de Marx y Engels fueron base parasu teoría (OLIVEIRA, 2011).Así, en la perspectiva marxista, la formación de la sociedad humana se basa en el trabajo y todos los fenómenos deben ser estudiados como procesos en permanente movimiento y transformación.

El hombre se constituye y se transforma al actuar sobre la naturaleza con su actividad y sus instrumentos, construyendo la historia y la cultura humana. En ese sentido, no podemos construir cualquier conocimiento a partir de la idea aparente, es imprescindible la interacción con el objeto y la comprensión de sus características constitutivas. En cambio, debemos delinear la evolución de los fenómenos, dado que es en su génesis y su movimiento que están las explicaciones de su historia y forma actual.

Los cambios individuales del sujeto tienen su raíz u origen en las condiciones sociales de la vida y la actividad colectiva, lo que ratifica el hombre como un ser social e histórico. Por consiguiente, no es la conciencia del hombre que determina las formas de vida, sino la vida que determina la conciencia. 
Una vez que el hombre es históricamente y socialmente determinado, debe ser comprendido por sus relaciones sociales y vínculos, así como por su inserción en una dicha sociedad o grupo cultural. Sin embargo, hay que considerar que el determinado momento histórico específico, el pensamiento y la acción humana existen en función de un dado momento, espacio y relaciones experimentadas.

La conciencia humana revela las determinaciones sociales e históricas del hombre - no directamente, de manera inmediata, porque no es así, mecánicamente, que se procesa la conciencia. Las mediaciones deben ser desvendadas, porque pasan a través de las formas de actividad y las relaciones sociales, por el significado asignado en ese proceso a la realidad en que todos los hombres viven. Es necesario conocer además del aparente, buscando la esencia de ese proceso, que revela el movimiento de constante transformación, a partir de la contradicción, entendida como un principio fundamental del movimiento de la realidad. (BOCK; FURTADO; TEIXEIRA, 2002, p. 120).

Así, a partir de los conceptos de la psicología sociohistórica y los postulados teóricos de Vygotsky, para conocer el hombre es necesario situarlo en un momento histórico, entender sus contradicciones y articularlas con en el materialismo histórico y dialéctico. Además, no nos olvidemos que los fenómenos sociales se desarrollande manera simultánea, interna y externamenteal individuo. De ahí que, tanto la subjetividad individual como la subjetividad social están implícitas en ese proceso.

La subjetividad debe ser comprendida como un sistema integrador del interno y externo, tanto en su dimensión social, como individual, que por su génesis también es social... La subjetividad no es interna y tampoco externa: ella supone otra representación teórica en la que el interno y el externo dejan de ser dimensiones excluyentes y se convierten en dimensiones constituyentes de una nueva calidad de ser: El subjetivo. Como ambos son dimensiones de la subjetividad (el interno y el externo) se integran y desintegran en una multitud de formas en el recogido de su desarrollo, en un proceso en lo cual lo que era interno puede convertirse en externo y viceversa. (GONZALEZ REY, 1997, p. 37-53).

Por tanto, la subjetividad individual es la constitución del sujeto, su historia y relaciones sociales en un sistema individual. Una vez que el sujeto vive determinadas relaciones sociales y experiencias en una cultura específica, constituirá y atribuirá significados y sentido a estas relaciones y expe- 
riencias que formarán su subjetividad individual. En cambio, la subjetividad social se refiere a los valores subjetivos tanto del grupo como del individuo que se articulan y se entrecruzan en los diferentes niveles de la esfera social.

Reflexionando a partir de las consideraciones hechas y de los estudios de la psicología sociohistórica (WERTSCH, 1988), no podemos entender o comprender al hombre sin conocer su mundo, el contexto histórico, social y cultural en que se encuentra inmerso. Los fenómenos del mundo psíquico no son inherentes del mundo psíquico, se van construyendo en la medida en que el sujeto vive las experiencias, actuando en el mundo y relacionándose con otros sujetos. En ese sentido, el contexto social y cultural es visto como un lugar donde nuestro mundo psicológico se constituye.

Sin embargo, comprender y analizar los fenómenos psíquicos y sociales, así como la adquisición y construcción de nuevas habilidades y competencias, implica en comprender e investigar la cultura actual y las cuestiones que la atraviesa.

Siendo así, la cultura, juntamente con los medios, tiene un efecto ideológico por la óptica materialista, refiriéndose a las formas asumidas por la existencia social y, consecuentemente, al contenido de las relaciones humanas y la forma como se representan estas relaciones. Esta cultura se hereda de generación en generación, a través de la forma de producción, del trabajo, y del lenguaje, es decir, el conocimiento, la cultura y el lenguaje tienen sus bases en la vida material y social.

En la perspectiva sociológica, las formas de producción material e intelectual son dominadas por una elite, que mantiene el control sobre los medios de producción intelectual. Así, las ideas dominantes son una extensión de las relaciones materiales dominantes. Por su parte, los pensadores y productores de ideas son los que "conducen" esas producciones.

La internalización de las formas culturales de comportamiento implica en la reconstrucción de la actividad psicológica basada en las operaciones con signos - los procesos psicológicos, tal y como aparecen en los animales realmente dejan de existir - son integrados en un sistema de comportamiento y son culturalmente reconstruidos y desarrollados para formar una nueva entidad psicológica. El uso de signos externos también es reconstruido radicalmente. Los cambios en las operaciones con los signos durante el desarrollo son similares a los que se producen en el lenguaje. Aspectos tanto en el discurso externo o comunicativo como en el discurso egocéntrico, se interiorizan convirtiéndose en la base para el discurso interior. (VYGOTSKY, 1995, p. 65). 
Como observamos en la cita anterior, la cultura es muy importante en el proceso del desarrollo humano. Los grupos culturales dónde los niños nacen, crecen y se desarrollan, producirán adultos que funcionarán psicológicamente de una manera particular, según los conceptos culturalmente construidos por este grupo específico. Para Vygotsky, la cultura proporciona a la persona un ambiente estructurado, donde todos los elementos son cargados de significados, y sus miembros están en constante movimiento, recreación y reinterpretación de la información, conceptos y significados, ratificando que el proceso del desarrollo humano está marcado por la inserción del sujeto en un determinado grupo cultural.

La internalización de las actividades socialmente arraigada e históricamente desarrolladas constituye el aspecto característico de la psicología humana; es la base del salto cualitativo de la psicología animal a la psicología humana. Hasta ahora se conoce solo un esquema de este proceso. (VYGOTSKY, 1995, p. 65).

Así, el hombre biológico se convierte en sociohistórico, a partir de un proceso de internacionalización de las actividades, comportamientos y signos culturalmente vividos y construidos. Por lo tanto, es importante tener en cuenta estos aspectos, principalmente en lo que toca al proceso de construcción del hombre como un ser sociohistórico y su desarrollo, así como el proceso de adquisición de nuevas competencias y habilidades que el mundo y entorno demandan.

\section{Alfabetización mediática y el pensamiento sociohistórico}

En este sentido, el pensamiento de Vygotsky es muy importante en la comprensión de la alfabetización formal o mediática, así como sus distintas formas de aprendizajes. La comprensión de la alfabetización y del proceso de construcción del conocimiento del individuoestá intrínsecamente vinculada al proceso de construcción del conocimiento mediático y digital, así como la adquisición de nuevas habilidades y competencias, es decir, la alfabetización mediática y la alfabetización formal son procesos análogos.

Históricamente, el término alfabetización mediática fue comentado en algunos textos de los estudios culturales que se destacaron al final de los años 50, el académico británico Richard Hoggart en su libroThe Uses of Literacy (1957), un texto autobiográfico en lo cual habla sobre la historia 
cultural del medio siglo XX y la influencia de los medios de comunicación en el Reino Unido. El autor comenta sobre la Alfabetización con los medios (Literacy) a través de la cultura popular, el consumo cotidiano de la cultura de masa y de los productos populares.

En la actualidad, tenemos la introducción de las TICs en los medios de comunicación y mediáticos, haciendo un gran cambio en los procesos de información, lectura, interpretación, análisis de los textos y del discurso (PEREZ TORNERO, 2000).

Hay un cambio cultural que no podemos denegar, una vez que la cultura atraviesa la construcción del conocimiento, la construcción de habilidades y competencias mediáticas también están insertadas en ese rol cultural, es decir, la utilización de los medios no es mecánica o meramente operativa, implica en construcción de conocimientos que estarán vinculados a la cultura y al proceso histórico y social.

Como comentamos anteriormente, el proceso de alfabetización mediática es análogo al proceso de alfabetización formal, ambas ocurren a lo largo de la vida e involucran todos los pasos que acompañan el desarrollo humano y sus especificidades, eso ratifica la naturaleza epistemológica de la alfabetización mediática la cual está directamente asociada a los procesos cognitivos y metacognitivos.

El entorno mediático es duplamente cultural, primero por estar sometido al proceso sociohistórico y, también, por estar implicado en la propia cultura de ese mismo entorno y sus demandas. La alfabetización mediática se refiere a la competencia y habilidad para el uso de nuevas tecnologías con numerosas similitudes con los procesos que se producen en el procedimiento de adquisición de lectura y escritura, aunque en la perspectiva mediática sea discutido y trabajado bajo otra lógica, pero está asociado a la dimensión educativa.

Por lo cual, no podemos reducir la alfabetización mediática al uso de las herramientas tecnológicas de manera operativa y técnica, invalidando su eficacia y acción social. Una vez que es un proceso de aprendizaje complejo, supone una inserción y transformación social que requieren un conjunto de conocimientos previos de distintas naturalezas (TEJEDOR; PERCEVAL, 2008).

No obstante, aunque sea un proceso personal e individual en su génesis, implica en una acción social y colectiva, una vez que se supone cambios en la sociedad. En este sentido, hay que considerar que paralelo 
a ese proceso, el contexto histórico y cultural actúan de manera directa en el desarrollo del sujeto, en los medios de comunicación y, concretamente, en la inclusión mediática del individuo en la Sociedad de la Información y del Conocimiento.

Trabajar a la luz del pensamiento de Vygotsky es abrir infinitas posibilidades al estudio y la investigación de la alfabetización mediática, visto que estamos inmersos en la cultura. Si pensamos de manera metafórica, la cultura representa una gran alfombra en "pachwork", construida a partir de las costumbres, valores y raíces culturales, mediadas e internalizadas por todos que componen la sociedad.

En ese sentido, podemos decir que alfabetizarse es utilizar en la vida, en la sociedad y en el mundo todos los conocimientos aprehendidos de manera crítica, constructiva y transformadora, respetando la cultura y la historia del entorno. Igualmente, la alfabetización mediática supone utilizar todas las herramientas ofrecidas por los medios desde una perspectivacrítica, actuando de manera participativa y colaborativa en la sociedad.

\section{Conclusión}

En ese artículo, hicimos una reflexión acerca de la relación existente entre la teoría sociocultural, el desarrollo humano vinculado al contexto cultural y la alfabetización mediática. A partir de estudios teóricos (BOCK; FURTADO; OLIVEIRA, 2011; TEIXEIRA, 2002; VYGOTSKY, 1995; HERNANDEZ, 2006), comprendemos que el término «alfabetización mediática” es más que una habilidad técnica y operativa, o un manejo sencillo de un equipo. Se trata de un concepto subjetivo que involucra aspectos cognitivos y metacognitivos, así como las dimensiones culturales y sociohistóricas.

Concluimos que la alfabetización mediática se refiere a la competencia y habilidad para el uso de nuevas tecnologías, similares en algunos aspectos, a los que se producen en el proceso de adquisición de lectura y escritura, aunque discutido y trabajado bajo otra lógica.

Por fin, podemos vincular los medios de comunicación y mediáticos al contexto cultural, y por supuesto al educacional, dado que es un proceso de aprendizaje y tiene una dimensión educativa. El proceso de alfabetización mediática aún requiere investigación y formación, sobretodo en el ámbito educativo, específicamente en los currículos escolares y en la formación docente. 


\section{Referencias Bibliográficas}

BOCK, A. M. B.; FURTADO, O.; TEIXEIRA, M. de L. T. Psicologias Uma Introdução ao Estudo de Psicologia. 13. ed. Sáo Paulo: Saraiva, 2002.

GONZÁLEZ REY, F.L. Epistemología cualitativa y subjetividad. 1. ed. São Paulo: EDUC, 1997.

HERNANDEZ, F. Tecnologias para transformar a educação. Porto Alegre: Artmed, 2006.

HOGGART, R. The Uses of Literacy. Aspects of Working-Class (1957). Londres: Penguin Group.

OLIVEIRA, M. K. de. Vygotsky:Aprendizado e Desenvolvimento - Um processo sócio-histórico. São Paulo: Editora Scipione, 2011.

PEREZ TORNERO, J. M. Comunicación y educación en la sociedad de la información. Barcelona: Paidós, 2000.

TEJEDOR, S. PERCEVAL, J.M. Los cinco grados de la comunicación en educación: Oral-gestual, escritura, audio, audiovisual, y ¿digital? Barcelona: Gabinete de Comunicación y educación de la Universidad Autónoma de Barcelona, 2008.

VYGOTSKY L. Pensamiento y Lenguaje. Barcelona: Paidós, 1995.

WERTSCH V. J. Vygotsky y la formación social de la mente. Barcelona: Paidós, 1988.

Recebido em: 26 ago. 2019.

Aceito em: 12 nov. 2019. 